\title{
«Patienten in den Fokus der Forschung stellen»
}

\author{
Interview: Felicitas Witte
}

Dr. med., Medizinjournalistin

«Zu wissen, was für wen wirkt» - das ist das Credo von Claudia Witt. Die Professorin leitet seit einem Jahr das Institut für komplementäre und integrative Medizin am Unispital Zürich, ihr Lehrstuhl ist der einzige dieser Art in der Schweiz. Eine pluralistische Gesundheitsversorgung ist ihr Ziel, basierend auf Evidenz und den Bedürfnissen der Patienten. Im Interview erzählt Witt, warum sie eine neue Forschungsrichtung, die Comparative Effectiveness Research, für so wichtig hält.

Sie leiten seit einem Jahr das Institut für komplementäre und integrative Medizin am Unispital Zürich.

Forscht es sich besser in der Schweiz als in Deutschland? Claudia Witt: Es forscht sich anders. Man kann in der Schweiz zwar keine Studien mit sehr grossen Patientenzahlen machen, weil sie dafür zu klein ist, aber dafür sehr innovative Forschung. Die Nähe von Unispital, Uni Zürich und ETH ist einfach eine super Voraussetzung, um mit Experten aller möglichen Fachrichtungen kooperieren zu können.

Warum finden Sie Komplementärmedizin so spannend? Mich hat schon am Anfang meines Medizinstudiums fasziniert, wie viele Menschen das anwenden und über Erfolge berichten, aber man wissenschaftlich viel zu wenig darüber weiss. Das Thema liess mich nicht mehr los. So habe ich erst in einer allgemeinmedizinischen Praxis gearbeitet, wo komplementäre Therapien angewendet wurden, und bin dann in die Epidemiologie der Charité gewechselt, um Forschung von der Pike auf zu lernen.

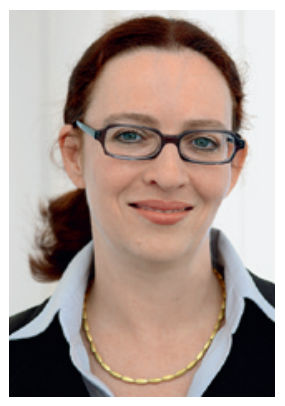

\section{Claudia Witt}

Nach Medizinstudium in Berlin und Bochum machte Claudia Witt einen Master of Business Administration und habilitierte sich im Bereich Sozialmedizin und Epidemiologie. In den USA lernte sie patientenzentrierte Forschung kennen. Von Mai 2011 bis April 2013 war die gebürtige Deutsche Präsidentin der International Society for Complementary Medicine Research. Durch ihre Beiträge für Forschung und Lehre hilft sie, über fachliche Grenzen hinaus das Verständnis im Hinblick auf die Bedeutung der integrativen und komplementären Medizin zu fördern, die den Menschen in seiner Ganzheitlichkeit ins Zentrum stellt.

\section{Einige Leute sehen in der komplementären Medizin} immer noch "Alternativ»-Medizin. Ärgert Sie das?

Ich sehe das viel gelassener, seitdem ich eine Studie mit internationalen Experten der komplementären und integrativen Medizin zum Thema Definitionen gemacht habe. Dabei kam raus, dass es den Befragten viel mehr darum ging, eine gute patientenzentrierte Medizin zu machen und die Terminologie eher sekundär war. Viele Patienten wenden komplementärmedizinische Massnahmen zusätzlich an, das wissen wir aus Studien. Wenn man gute Medizin machen möchte, geht es nicht um ein Entweder-oder, sondern darum, die beste Therapie für den einzelnen Patienten zu finden.

Welchen Stellenwert sehen Sie in der Komplementärmedizin innerhalb der sogenannten Schulmedizin? Hierbei müssen Sie mir erst sagen, was Sie mit Komplementärmedizin meinen. Das Spektrum ist sehr breit - von chinesischer Medizin über Entspannungstechniken, Osteopathie oder Nahrungsergänzungsmittel. Einige komplementärmedizinische Verfahren könnten einen guten Beitrag in einem umfassenden Therapiekonzept leisten. So zum Beispiel das neue Angebot unseres Instituts in Zusammenarbeit mit der Klinik für Radio-Onkologie: Mit Patienten, die eine Strahlentherapie erhalten und aktiv ihre Widerstandsfähigkeit stärken möchten, erarbeiten wir ein individuelles Behandlungsprogramm aus der Mind Body Medicine, zu dem Entspannung und Bewegung gehören. Treten im Verlauf der Therapie Nebenwirkungen auf, etwa Fatigue, bieten wir zum Beispiel Akupunktur oder Phytotherapie an. Ähnliche Möglichkeiten gibt es natürlich auch in anderen Fachbereichen. 


\section{Bekommen Sie «Gegenwind» von klassischen Schulmedizinern?}

Am Unispital in Zürich klappt die Zusammenarbeit prima, ich bin sehr freundlich aufgenommen worden. Ich erlebe natürlich immer mal wieder Skepsis aber das bin ich gewohnt. Doch auch mit skeptischen Kollegen lässt sich eine gemeinsame Ebene finden. Denn ich bin ja nicht nur Komplementärmedizinerin, sondern auch eine gut ausgebildete Wissenschaftlerin und Managerin.

\section{Sie sagten in unserem Telefongespräch als Erstes, Ihre Bilanz nach dem ersten Jahr sei sehr positiv. Was genau meinten Sie damit?}

Ich bin gut in der Schweiz angekommen und würde mich immer wieder für diese Stelle entscheiden. Mein Institut gehört ganz selbstverständlich zum Unispital, und wir sind mit unserer Poliklinik voll integriert. Dadurch haben sich spannende Kooperationen entwickelt, etwa Akupunkturforschung mit dem Zentrum für Zahnmedizin. Das finde ich auch gut an der Schweiz: Hier legt man viel Wert auf Kooperation, das fördert die Vernetzung und in der Wissenschaft kommen wir damit schneller zu Ergebnissen.

\section{Keine Kritik?}

Doch. Trotz des grossen Interesses der Bevölkerung an der Komplementärmedizin wurde bisher kein Forschungsschwerpunkt eingerichtet. Die Amerikaner sind hier weiter. Nachdem klar war, dass viele Menschen komplementärmedizinische Massnahmen in Anspruch nehmen, wurde an den National Institutes of Health ein eigenes Institut dafür eingerichtet und Forschungsgelder zur Verfügung gestellt. Und es wurde Wert darauf gelegt, junge Forscher in diesem Bereich speziell fortzubilden. Das wäre hier auch sinnvoll.

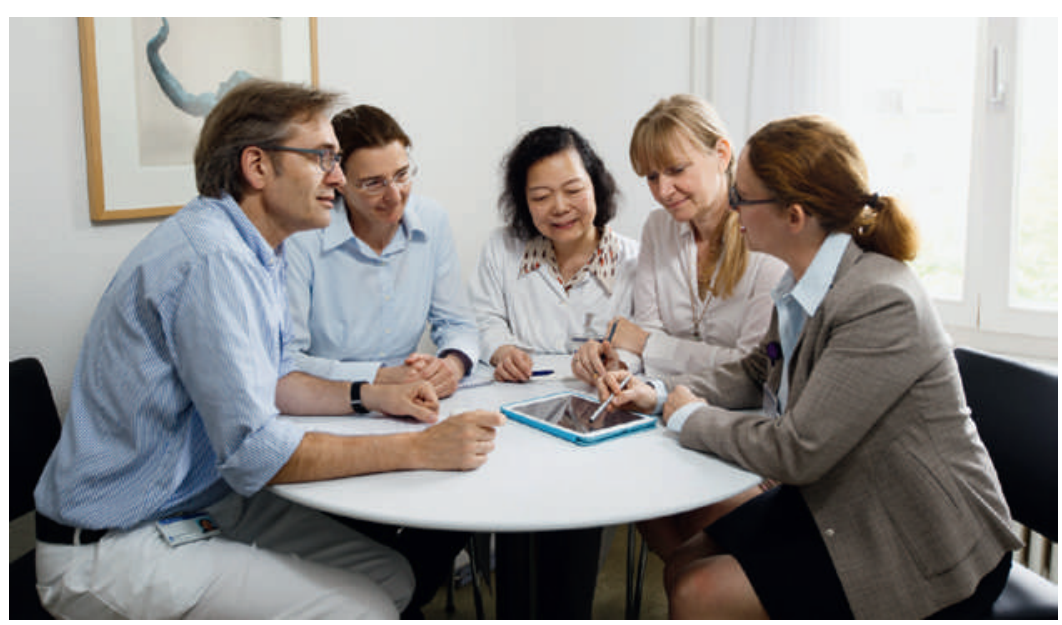

Claudia Witt legt grossen Wert auf die Zusammenarbeit mit Experten aus den verschiedensten Fachrichtungen.

\section{Aus den USA nahmen Sie noch etwas anderes mit, Comparative Effectiveness Research, kurz CER. Was ist das und wofür soll das gut sein?}

CER bedeutet, dass man untersucht, ob eine Therapie nicht nur bei selektierten Studienpatienten wirkt, sondern auch unter Alltagsbedingungen bei «ganz normalen" Patienten und wenn sie weniger standardisiert ist. Das finde ich sehr wichtig. Denn wir müssen wissen, ob eine Behandlung auch im Alltag wirkt.

\section{Für Statistiker gilt die RCT als «Goldstandard», um die Wirksamkeit von Behandlungen nachzuweisen. Hat die RCT ausgedient?}

Nein, sie ist nach wie vor für bestimmte Studienfragen unverzichtbar. Randomisierte Studien gehören auch zur CER. Aber in der CER sollen unbedingt die "typischen" Patienten eingeschlossen werden und nicht nur eine Gruppe hochselektierter Patienten. Als Epidemiologin sehe ich Studienergebnisse oft kritisch, denn man kann sie nur selten 1:1 in die Praxis übertragen. Man muss sich die Ein- und Ausschlusskriterien genau anschauen. Erfüllt der Patient diese nicht, lassen sich die Ergebnisse auch nicht direkt übertragen. Manche Medikamente wirkten bei einer klar definierten Patientengruppe unter Studienbedingungen sehr gut. Wurden sie dann in der Praxis eingesetzt, in der Patienten diverse andere Medikamente gleichzeitig nahmen und noch unter anderen Krankheiten litten, verschlechterte sich oft die Wirkung und es kam häufiger zu Nebenwirkungen. Stimmen die Ein- und Ausschlusskriterien mit dem jeweiligen Patienten nicht überein, bewegt man sich schnell im evidenzfreien Bereich.

\section{Studien müssen doch standardisiert aufgebaut sein und klar definierte Patientengruppen umfassen, damit man zu einem möglichst validen Ergebnis kommt. Wie passt das zu CER?}

Das Design einer Studie hängt primär von der Frage ab, die man beantworten will. Möchte ich wissen, ob ich genau in diese Akupunkturpunkte stechen muss, dann muss ich Akupunktur mit Scheinakupunktur in einer klassischen RCT vergleichen. Die Studie sagt mir dann aber nicht, ob Akupunktur auch im Alltag wirkt. Will ich herausfinden, ob bei einer Schmerztherapie ein komplexes Therapiekonzept mit Akupunktur besser wirkt als ohne, plane ich eine randomisierte pragmatische Studie: Eine Gruppe erhält Akupunktur zusätzlich zur konventionellen Schmerztherapie, die andere nicht. In diese Studie würde ich möglichst viele heterogene Patienten einschliessen, um später auch Subgruppen auswerten zu können. Dann hat man die Möglichkeit, Gesamtaussagen über die Ge- 
samtgruppe, aber auch Detailaussagen zu Untergruppen zu treffen, zum Beispiel ob Männer anders reagieren als Frauen oder Patienten mit starken

\section{"Mit anderen Zentren des Unispitals Zürich}

haben sich spannende Kooperationen entwickelt.»

Schmerzen anders als diejenigen mit weniger starken Schmerzen. In pragmatischen Studien können wir demzufolge nicht zwischen akupunkturspezifischen und unspezifischen Effekten unterscheiden. Dies ist nur im direkten Vergleich von Akupunktur und Scheinakupunktur möglich. Es kommt also immer auf die Fragestellung an: Man kann nicht die Frage nach dem akupunkturpunktspezifischen Effekt und der Effektivität in der Routineversorgung mit derselben Art von Studie beantworten.

\section{Die US-Amerikaner sind bei CER mal wieder Vorreiter. Woran hapert es in Europa?}

Die CER entstand dadurch, dass Entscheidungsträger in Krankenversicherungen und Politik frustriert waren, weil viele Ergebnisse nicht in den Alltag übertragbar sind. Daraufhin stellt die Regierung seit 2009 Forschungsgelder bereit und gründete 2010 das $\mathrm{Pa}$ tient-Centered Outcomes Institute (PCORI). Durch neue Forschungsgelder gab es einen klaren Anreiz für die Forscher, sich mit dem Thema auseinanderzusetzen und sich auch fortzubilden. Im Moment führe ich in den USA gerade ein Projekt durch, welches durch die NIH gefördert wird. Wir entwickeln eine Skala, mit der man in systematischen Reviews erkennen kann, ob die zusammengefassten Studien eher Evidenz aus einem idealisierten Studiensetting bieten oder aus dem Alltag.

Ist nicht die Gefahr für einen Bias bei CER-Studien viel grösser als bei Studien unter «idealen» Bedingungen? Das ist definitiv so. Aber in der CER geht es ja genau darum, die richtige Balance zwischen Biasrisiko und Relevanz der Ergebnisse für die Endnutzer zu finden. Ausserdem entwickelt sich die CER ständig weiter und ermöglicht es, auch Brücken zu bilden. Wir haben gerade ein Manuskript in einem Sonderheft der Zeitschrift Science zur Chinesischen Medizin publiziert, in dem wir Vorschläge gemacht haben, wie man CER mit modernen Messparametern wie z.B. Proteonomics und Genomics kombinieren kann.

\section{Sind Ihre Kollegen hierzulande offen für CER?}

Dr. med. Felicitas Witte

Seefeldstrasse 285

CH-80o8 Zürich

felicitas.witte[at]icloud.com
Entspannungs-Apps den Patienten helfen können. Die Studien führe ich aber zurzeit noch in Deutschland durch, weil ich sie erstens vor meinem Wechsel begonnen habe und weil es zweitens einfacher ist, grössere Patientengruppen einzuschliessen. Am Unispital gab es bisher noch wenig Berührung mit dem Thema, das wird sich aber durch den Ausbau der Versorgungsforschung und die Diskussionen in diesem Zusammenhang sicherlich ändern.

Welche Rolle spielt CER in der Komplementärmedizin? Eine grosse, denn es geht hier häufig um versorgungsrelevante Fragen und CER kann diese gut beantworten. Ich bin ein gutes Beispiel dafür, denn letztendlich haben meine versorgungsrelevanten Fragen dazu geführt, dass ich mich mit der Methodik der CER eingehend beschäftige. Und das müssen wir in der Medizin, denn Fragen nach dem Nutzen einer Therapie unter «Normalbedingungen» werden immer wichtiger, gerade bei den neuen, teuren Medikamenten.

\section{Wie könnte in der Schweiz ein besserer Schwerpunkt auf CER gelegt werden?}

Das BAG plant, in den Ausbau der Versorgungsforschung zu investieren. Die CER ist ein Teil der Versorgungsforschung, hat aber stark den Anspruch, Stakeholder in die Forschung einzubinden, das sind Interessenvertreter wie Patienten, Ärzte, Politiker und Krankenkassen. CER fordert, Stakeholder bei der Priorisierung der Forschungsschwerpunkte, bei der Planung der Studien und bei der Interpretation der Studienergebnisse einzubeziehen. Gerade die Schweiz mit ihrem sehr kooperativen demokratischen Ansatz könnte sich noch mehr engagieren, Stakeholder in die Forschung einzubinden.

\section{Warum sind dabei die Patienten so wichtig?}

Weil sie die Forschung voranbringen. Wir haben z.B. in einer Studie Akupressur im Rahmen einer App auf dem Handy bei Dysmenorrhoe untersucht, und zwar nur, weil uns Patientinnen darauf brachten.

\section{Käme jetzt eine Fee: Was würden Sie sich wünschen?} Dass wir wirklich die Patienten ins Zentrum stellen und uns gemeinsam bemühen, dass sie die beste Versorgung bekommen. Zudem sollten wir unbedingt berücksichtigen, dass die medizinische Versorgung nur ein Faktor ist, der zur Gesundheit des Einzelnen und der Bevölkerung beiträgt. So haben natürlich die Genetik und das soziale Umfeld Einfluss, aber auch der Lebensstil. Mit Mind Body Medicine und Naturheilkunde können wir dazu beitragen, dass Leute ihren Lebensstil optimieren und so länger gesund bleiben. 